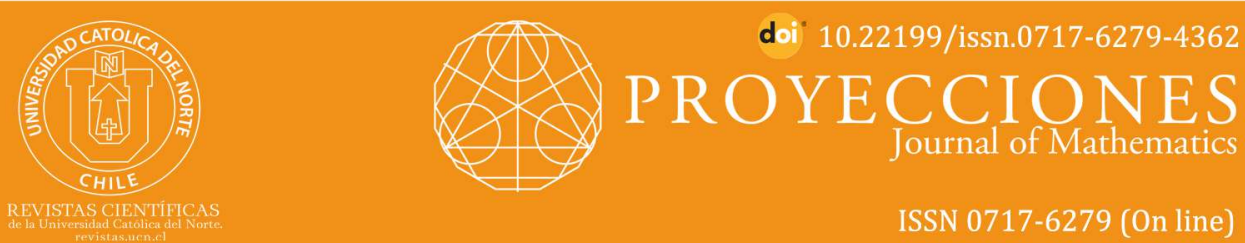

\title{
Square root stress-sum index for graphs
}

K. B. Mahesh ${ }^{1}$

R. Rajendra² (i) orcid.org/0000-0003-3562-3147

P. Siva Kota Reddy ${ }^{3}$ orcid.org/0000-0003-4033-8148

${ }^{1}$ Dr. P. Dayananda Pai- P. Sathisha Pai, Government First Grade College, Mangalore, Carstreet. Mangalore, KA, India. mathsmahesh@gmail.com

${ }^{2}$ Mangalore University, Dept. of Mathematics. Mangalore, KA, India.

rrajendrar@gmail.com

${ }^{3}$ JSS Science and Technology University, Sri Jayachamarajendra College of Engineering, Dept. of Mathematics. Mysurui, KA, India. pskreddy@jssstuniv.in

Received: 2020/07/26 | Accepted: 2021/01/15

\section{Abstract:}

The stress of a vertex is a centrality index, which has been introduced by Shimbel (1953). The stress of a vertex in a graph is the number of geodesics (shortest paths) passing through it. In this paper, we introduce a new topological index for graphs called square root stress-sum index using stresses of vertices. Further, we establish some inequalities, obtain some results and compute square root stress-sum index for some standard graphs.

Keywords: Geodesic; Stress of a vertex; Topological index.

MSC (2020): 05C09, 05C38.

\section{Cite this article as (IEEE citation style):}

K. B. Mahesh, R. Rajendra, and P. S. K. Reddy, "Square root stress-sum index for graphs", Proyecciones (Antofagasta, On line), vol. 40, no. 4, pp. 927-936, 2021, doi: 10.22199/issn.0717-6279-4362

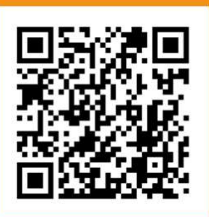

Article copyright: (C) 2021 K. B. Mahesh, R. Rajendra and P. Siva Kota Reddy. This is an open access article distributed under the terms of the Creative Commons License, which permits unrestricted use and distribution provided the original author and source are credited.

(cc) BY 


\section{Introduction}

For standard terminology and notion in graph theory, we follow the textbook of Harary [3]. The non-standard will be given in this paper as and when required.

Let $G=(V, E)$ be a graph (finite and undirected). The distance between two vertices $u$ and $v$ in $G$, denoted by $d(u, v)$ is the number of edges in a shortest path (also called a graph geodesic) connecting them. We say that a graph geodesic $P$ is passing through a vertex $v$ in $G$ if $v$ is an internal vertex of $P$ (i.e., $v$ is a vertex in $P$, but not an end vertex of $P$ ). $v$ in $G$, $g(u, v)$ denotes the number of geodesics whose end vertices are $u$ and $v$. The degree of a vertex $v$ in $G$ is denoted by $d(v)$.

The concept of stress of a node (vertex) in a network (graph) has been introduced by Shimbel as a centrality measure in 1953 [6]. This centrality measure has applications in biology, sociology, psychology, etc., (See [4, $5])$. The stress of a vertex $v$ in a graph $G$, denoted by $\operatorname{str}(v)$, is the number of geodesics passing through it. We denote the maximum stress among all the vertices of $G$ by $\Theta_{G}$ and minimum stress among all the vertices of $G$ by $\theta_{G}$. Further, the concepts of stress number of a graph and stress regular graphs have been studied by K. Bhargava, N.N. Dattatreya, and R. Rajendra in their paper [1].

The reciprocal sum-connectivity index of a graph (see [2]) is defined as

$$
R S C(G)=\sum_{u v \in E(G)} \sqrt{d(u)+d(v)}
$$

Motivated by the identity sqrt, in this paper, we introduce a new topological index called square root stress-sum index using stresses on vertices. Further, we establish some inequalities, obtain some results and compute stress-sum index for some standard graphs.

\section{Square Root Stress-Sum Index for Graphs}

Definition 2.1. The square root stress-sum index $\mathcal{S R S}(G)$ of a simple graph $G$ is defined as

$$
\mathcal{S R S}(G)=\sum_{u v \in E(G)} \sqrt{(u)+(v)}
$$


Observation: From the Definition 2.1, it follows that, for any graph $G$,

$$
2 m \sqrt{\theta_{G}} \leq \mathcal{S R S}(G) \leq 2 m \sqrt{\Theta_{G}},
$$

where $m$ is the number of edges in $G$.

Example 2.2. Consider the graph $G$ given in Figure 2.1.

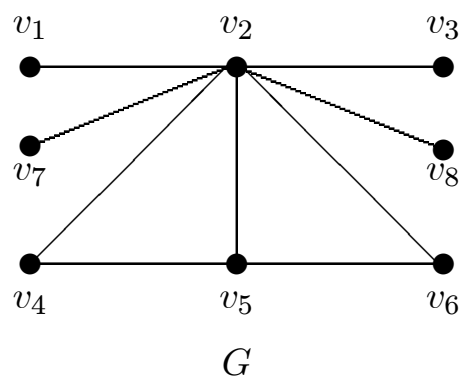

Figure 2.1: A graph $G$

The stresses of the vertices of $G$ are as follows:

$$
\begin{aligned}
& \operatorname{str}\left(v_{1}\right)=\operatorname{str}\left(v_{3}\right)=\operatorname{str}\left(v_{7}\right)=\operatorname{str}\left(v_{8}\right)=0, \\
& \operatorname{str}\left(v_{2}\right)=19 \text {, } \\
& \operatorname{str}\left(v_{5}\right)=1 \text {, } \\
& \operatorname{str}\left(v_{4}\right)=\operatorname{str}\left(v_{6}\right)=0 . \\
& \text { The stress-sum index of } G \text { is: } \\
& \mathcal{S R S}(G)=\sqrt{\left(v_{2}\right)+\left(v_{1}\right)}+\sqrt{\left(v_{2}\right)+\left(v_{3}\right)}+\sqrt{\left(v_{2}\right)+\left(v_{7}\right)} \\
& +\sqrt{\left(v_{2}\right)+\left(v_{8}\right)}+\sqrt{\left(v_{2}\right)+\left(v_{4}\right)}+\sqrt{\left(v_{2}\right)+\left(v_{5}\right)} \\
& +\sqrt{\left(v_{2}\right)+\left(v_{6}\right)}+\sqrt{\left(v_{4}\right)+\left(v_{5}\right)}+\sqrt{\left(v_{5}\right)+\left(v_{6}\right)} \\
& =\sqrt{19+0}+\sqrt{19+0}+\sqrt{19+0}+\sqrt{19+0}+\sqrt{19+0}+\sqrt{19+1} \\
& +\sqrt{19+0}+\sqrt{0+1}+\sqrt{1+0} \\
& =2+6 \sqrt{19}+\sqrt{20} \text {. }
\end{aligned}
$$

Proposition 2.3. Let $N$ be the number of geodesics of length $\geq 2$ in a graph $G$. Then

$$
0 \leq \mathcal{S R S}(G) \leq \sqrt{2 N}(|E|-t),
$$

where $t$ is the number of edges with end vertices having zero stress in $G$. 
Proof. If $N$ is the number of all geodesics of length $\geq 2$ in a graph $G$, then by the definition of stress of a vertex, for any vertex $v$ in $G$, $0 \leq \operatorname{str}(v) \leq N$. Hence by the Definition 2.1, we have

$$
0 \leq \mathcal{S R S}(G) \leq \sqrt{2 N}(|E|-t),
$$

where $t$ is the number of edges with end vertices having zero stress in $G$.

Corollary 2.4. If there is no geodesic of length $\geq 2$ in a graph $G$, then $\mathcal{S R S}(G)=0$. Moreover, for a complete graph $K_{n}, \mathcal{S R S}\left(K_{n}\right)=0$.

Proof. If there is no geodesic of length $\geq 2$ in a graph $G$, then $N=0$. Hence, by the Proposition 2.3, we have $\mathcal{S R S}(G)=0$.

In $K_{n}$, there is no geodesic of length $\geq 2$ and so $\mathcal{S R S}\left(K_{n}\right)=0$.

Theorem 2.5. For a graph $G, \mathcal{S R S}(G)=0$ if and only if neighbours of every vertex induce a complete subgraph of $G$.

Proof. Suppose that $\mathcal{S R S}(G)=0$. Then by the Definition 2.1(Eq.srs), $\sqrt{(u)+(v)}=0, \forall u v \in E(G)$ and so $(u)+(v)=0, \forall u v \in E(G)$. Hence $(v)=0, \forall v \in V(G)$. Let $v \in V(G)$. We need to show that neighbours of $v$ induce a complete subgraph of $G$. If $v$ is a pendant vertex, then there is nothing to prove. Suppose that $v$ is not a pendant vertex. We claim that any two neighbouring vertices are adjacent in $G$. If there are two neighbours $u$ and $w$ of $v$ that are not adjacent in $G$, then $u v w$ is a graph geodesic passing through $v$, which implies $(v) \geq 1$, a contradiction. Hence our claim holds. Thus neighbours of $v$ induce a complete subgraph of $G$. Since $v$ is arbitrary in $V(G)$, the neighbours of every vertex induce a complete subgraph of $G$.

Conversely, suppose that neighbours of every vertex in $G$ induce a complete subgraph of $G$. Let $v \in V(G)$. Since neighbours of $v$ induce a complete subgraph of $G$, any two neighbouring vertices are adjacent and so there is no geodesic of length $\geq 2$ passing through $v$. Since $v$ is an arbitrary vertex in $G$, by the Corollary 2.4, it follows that $\mathcal{S R S}(G)=0$.

Proposition 2.6. For the complete bipartite $K_{r, s}$,

$$
\mathcal{S R S}\left(K_{r, s}\right)=\frac{r s}{\sqrt{2}} \sqrt{s(s-1)+r(r-1)} .
$$


Proof. Let $V_{1}=\left\{v_{1}, \ldots, v_{r}\right\}$ and $V_{2}=\left\{u_{1}, \ldots, u_{s}\right\}$ be the partite sets of $K_{r, s}$. We have,

$$
\left(v_{i}\right)=\frac{s(s-1)}{2} \text { for } 1 \leq i \leq r
$$

and

$$
\left(u_{j}\right)=\frac{r(r-1)}{2} \text { for } 1 \leq j \leq s .
$$

Using 2.5 and 2.6 in the Definition 2.1, we have

$$
\begin{aligned}
\mathcal{S R S}\left(K_{r, s}\right) & =\sum_{u v \in E(G)} \sqrt{(u)+(v)} \\
& =\sum_{1 \leq i \leq r, 1 \leq j \leq r} \sqrt{\left(v_{i}\right)+\left(u_{j}\right)} \\
& =\sum_{1 \leq i \leq r, 1 \leq j \leq s}\left[\sqrt{\frac{s(s-1)}{2}+\frac{r(r-1)}{2}}\right] \\
& =r s\left[\sqrt{\frac{s(s-1)}{2}+\frac{r(r-1)}{2}}\right] \\
& =\frac{r s}{\sqrt{2}} \sqrt{s(s-1)+r(r-1)} .
\end{aligned}
$$

Proposition 2.7. If $G=(V, E)$ is a $k$-stress regular graph, then

$$
\mathcal{S R S}(G)=\sqrt{2 k}|E| .
$$

Proof. Suppose that $G$ is a $k$-stress regular graph. Then

$$
(v)=k \text { for all } v \in V(G) \text {. }
$$

By the Definition 2.1, we have

$$
\begin{aligned}
\mathcal{S R S}(G) & =\sum_{u v \in E(G)} \sqrt{(u)+(v)} \\
& =\sum_{u v \in E(G)} \sqrt{k+k} \\
& =\sqrt{2 k}|E| .
\end{aligned}
$$

Corollary 2.8. For a cycle $C_{n}$,

$$
\mathcal{S R S}\left(C_{n}\right)=\begin{array}{ll}
\frac{n(n-1)(n-3)}{4}, & \text { if } n \text { is odd; } \\
\frac{n^{2}(n-2)}{4}, & \text { if } n \text { is even. }
\end{array}
$$


Proof. For any vertex $v$ in $C_{n}$, we have,

$$
(v)=\begin{array}{ll}
\frac{(n-1)(n-3)}{8}, & \text { if } n \text { is odd; } \\
\frac{n(n-2)}{8}, & \text { if } n \text { is even. }
\end{array}
$$

Hence $C_{n}$ is

$$
\begin{array}{ll}
\frac{(n-1)(n-3)}{8} \text {-stress regular, } & \text { if } n \text { is odd; } \\
\frac{n(n-2)}{8} \text {-stress regular, } & \text { if } n \text { is even. }
\end{array}
$$

Since $C_{n}$ has $n$ vertices and $n$ edges, by the Proposition 2.7, we have

$$
\begin{aligned}
& \mathcal{S R S}\left(C_{n}\right)=n \times \sqrt{2 \cdot \frac{(n-1)(n-3)}{8}}, \text { if } n \text { is odd; }
\end{aligned}
$$

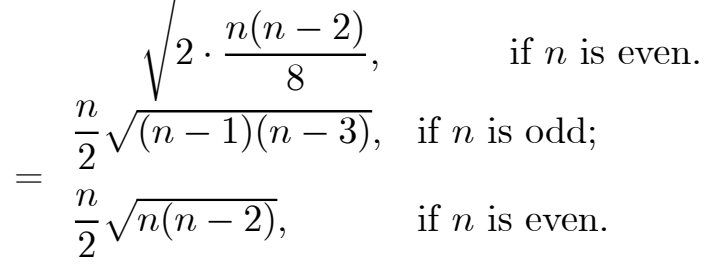

Proposition 2.9. Let $T$ be a tree on $n$ vertices. Then

$$
\begin{gathered}
\mathcal{S R S}(T)=\sum_{u v \in J} \sqrt{\sum_{1 \leq i<j \leq m(u)}\left|C_{i}^{u}\right|\left|C_{j}^{u}\right|+\sum_{1 \leq i<j \leq m(v)}\left|C_{i}^{v}\right|\left|C_{j}^{v}\right|} \\
+\sum_{w \in Q} \sqrt{\sum_{1 \leq i<j \leq m(w)}\left|C_{i}^{w}\right|\left|C_{j}^{w}\right|} .
\end{gathered}
$$

where $J$ is the set of internal(non-pendant) edges in $T, Q$ denotes the set of all vertices adjacent to pendant vertices in $T$, and the sets $C_{1}^{v}, \ldots, C_{m}^{v}$ denotes the vertex sets of the components of $T-v$ for an internal vertex $v$ of degree $m=m(v)$.

Proof. We know that a pendant vertex in $T$ has zero stress. Let $v$ be an internal vertex of $T$ of degree $m=m(v)$. Let $C_{1}^{v}, \ldots, C_{m}^{v}$ be the components of $T-v$. Since there is only one path between any two vertices in a tree, it follows that,

$$
\operatorname{str}(v)=\sum_{1 \leq i<j \leq m}\left|C_{i}^{v}\right|\left|C_{j}^{v}\right|
$$

Let $J$ denotes the set of internal(non-pendant) edges, and $P$ denotes pendant edges and $Q$ denotes the set of all vertices adjacent to pendant vertices in $T$. Then using Qstress in the Definition 2.1, we have 


$$
\begin{array}{ll}
\mathcal{S R S}(T)= & \sum_{u v \in J} \sqrt{(u)+(v)}+\sum_{u v \in P} \sqrt{(u)+(v)} \\
= & \sum_{u v \in J} \sqrt{(u)+(v)}+\sum_{w \in Q} \sqrt{(w)} \\
= & \sum_{u v \in J} \sqrt{\sum_{1 \leq i<j \leq m(u)}\left|C_{i}^{u}\right|\left|C_{j}^{u}\right|+\sum_{1 \leq i<j \leq m(v)}\left|C_{i}^{v}\right|\left|C_{j}^{v}\right|} \\
& \\
& +\sum_{w \in Q} \sqrt{\sum_{1 \leq i<j \leq m(w)}\left|C_{i}^{w}\right|\left|C_{j}^{w}\right|} .
\end{array}
$$

Corollary 2.10. For the path $P_{n}$ on $n$ vertices

$$
\mathcal{S R S}\left(P_{n}\right)=\sum_{i=1}^{n-1} \sqrt{2 i n-2 i^{2}-n}
$$

Proof. The proof of this corollary follows by above Proposition 2.9. We follow the proof of the Proposition 2.9 to compute the index. Let $P_{n}$ be the path with vertex sequence $v_{1}, v_{2}, \ldots, v_{n}$ (shown in Figure 2.2).

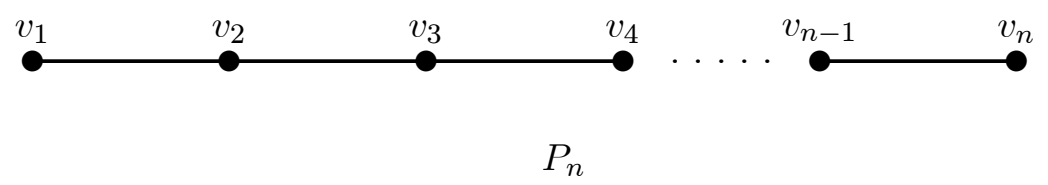

Figure 2.2: The path $P_{n}$ on $n$ vertices.

We have,

$$
\operatorname{str}\left(v_{i}\right)=(i-1)(n-i), \quad 1 \leq i \leq n .
$$

Then

$$
\begin{aligned}
\mathcal{S R S}\left(P_{n}\right) & =\sum_{u v \in E\left(P_{n}\right) \sqrt{(u)+(v)}} \\
& =\sum_{i=1}^{n-1} \sqrt{\left(v_{i}\right)+\left(v_{i+1}\right)} \\
& =\sum_{i=1}^{n-1} \sqrt{(i-1)(n-i)}+(i)(n-i-1) \\
& =\sum_{i=1}^{n-1} \sqrt{2 i n-2 i^{2}-n} .
\end{aligned}
$$

Proposition 2.11. Let $W d(n, m)$ denotes the windmill graph constructed for $n \geq 2$ and $m \geq 2$ by joining $m$ copies of the complete graph $K_{n}$ at a shared universal vertex $v$ (a universal vertex of a graph is a vertex that is adjacent to all other vertices of the graph). Then

$$
\mathcal{S R S}(W d(n, m))=m(n-1)^{2} \sqrt{\frac{m(m-1)}{2}} .
$$

Hence, for the friendship graph $F_{k}$ on $2 k+1$ vertices,

$$
\mathcal{S R S}\left(F_{k}\right)=4 k \sqrt{\frac{k(k-1)}{2}} .
$$


Proof. Clearly the stress of any vertex other than universal vertex is zero in $W d(n, m)$, because neighbours of that vertex induces a complete subgraph of $W d(n, m)$. Also, since there are $m$ copies of $K_{n}$ in $W d(n, m)$ and their vertices are adjacent to $v$, it follows that, the only geodesics passing through $v$ are of length 2 only. So, $(v)=\frac{m(m-1)(n-1)^{2}}{2}$. Note that there are $m(n-1)$ edges incident on $v$ and the edges that are not incident on $v$ have end vertices of stress zero. Hence by the Definition 2.1, we have

$$
\begin{aligned}
\mathcal{S R S}(W d(n, m)) & =m(n-1) \sqrt{(v)} \\
& =m(n-1) \sqrt{\frac{m(m-1)(n-1)^{2}}{2}} \\
& =m(n-1)^{2} \sqrt{\frac{m(m-1)}{2}} .
\end{aligned}
$$

Since the friendship graph $F_{k}$ on $2 k+1$ vertices is nothing but $W d(3, k)$, it follows that

$$
\mathcal{S} \mathcal{R} \mathcal{S}\left(F_{k}\right)=4 k \sqrt{\frac{k(k-1)}{2}} .
$$

Proposition 2.12. Let $W_{n}$ denote the wheel graph constructed on $n \geq 4$ vertices. Then

$$
\mathcal{S R S}\left(W_{n}\right)=(n-1) \times \sqrt{\frac{(5 n-6)(n-4)}{8}}+\sqrt{\frac{(n-2)(n-4)}{4}}, \quad \text { if } n \text { is even; }
$$

Proof. In $W_{n}$ with $n \geq 4$, there are $(n-1)$ peripheral vertices and one central vertex, say $v$. It is easy to see that

$$
(v)=\frac{(n-1)(n-4)}{2}
$$

Let $p$ be a peripheral vertex. Since $v$ is adjacent to all the peripheral vertices in $W_{n}$, there is no geodesic passing through $p$ and containing $v$. Hence contributing vertices for $(p)$ are the remaining peripheral vertices. So, by denoting the cycle $W_{n}-p$ (on $n-1$ vertices) by $C_{n-1}$, we have 


$$
\begin{aligned}
W_{n}(p)= & W_{n-v}(p) \\
= & C_{n-1}(p) \\
= & \frac{(n-2)(n-4)}{8}, \quad \text { if } n-1 \text { is odd; } \\
& \frac{(n-1)(n-3)}{8}, \quad \text { if } n-1 \text { is even, } \\
= & \frac{(n-2)(n-4)}{8}, \quad \text { if } n \text { is even; } \\
& \frac{(n-1)(n-3)}{8}, \quad \text { if } n \text { is odd. }
\end{aligned}
$$

Let us denote the set of all radial edges in $W_{n}$ by $R$, and the set of all peripheral edges by $Q$. Note that there are $(n-1)$ radial edges and $(n-1)$ peripheral edges in $W_{n}$. Using cntr and peri in the Definition 2.1, we have

$$
\begin{aligned}
& \mathcal{S R S}\left(W_{n}\right)=\sum_{x y \in R} \sqrt{(x)+(y)}+\sum_{x y \in Q} \sqrt{(x)+(y)} \\
& =(n-1) \sqrt{(v)+(p)+(n-1) \sqrt{2 \cdot(p)}}\left[\begin{array}{l}
\sqrt{\frac{(n-1)(n-4)}{2}+\frac{(n-2)(n-4)}{8}}, \text { if } n \text { is even; } \\
=(n-1)
\end{array} \quad \text { if } n\right. \text { is odd. } \\
& +\sqrt{\frac{(n-1)(n-4)}{2}+\frac{(n-1)(n-3)(n-4)}{8}}, \text { if } n \text { is even; } \\
& =(n-1) \times \sqrt{\frac{(5 n-6)(n-4)}{8}+\sqrt{\frac{(n-2)(n-4)}{4}}, \text { if } n \text { is odd. } n \text { is even; }} \\
& \sqrt{\frac{(n-1)(5 n-19)}{8}}+\sqrt{\frac{(n-1)(n-3)}{4}}, \text { if } n \text { is odd. }
\end{aligned}
$$

\section{Conclusion}

Based on vertex degrees, a large number of topological indices have been defined and studied by several authors. We have introduced a new topological index for graphs called square root stress-sum index using stresses of vertices. Further, we established some inequalities, obtained some results and computed the stress-sum index for some standard graphs. The characterizations between properties of graphs and this index will be reported 
in a subsequent paper.

\section{Acknowledgment}

The authors would like to thank the anonymous referee for his valuable comments which helped to improve the manuscript.

\section{References}

[1] K. Bhargava, N.N. Dattatreya, and R. Rajendra, "On stress of a vertex in a graph", Palestine journal of mathematics, accepted for publication.

[2] K. C. Das, I. Gutman, I. Milovanović, E. Milovanović, and B. Furtula, "Degree-based energies of graphs", Linear algebra and its applications, vol. 554, pp. 185-204, 2018, doi: 10.1016/j.laa.2018.05.027

[3] F. Harary, Graph theory. Reading, MA: Addison Wesley, 1972.

[4] M. Indhumathy, S. Arumugam, V. Baths, and T. Singh, "Graph theoretic concepts in the study of biological networks", in Applied analysis in biological and physical sciences, J. Cushing, M. Saleem, H. Srivastava, M. Khan, and M. Merajuddin, Eds. New Delhi: Springer, 2016, pp. 187-200, doi: 10.1007/978-81-322-3640-5_11

[5] P. Shannon, A. Markiel, O. Ozier, N.S. Baliga, J.T. Wang, D. Ramage, N. Amin, B. Schwikowski, and T. Idekar, "Cytoscape: a software environment for integrated models of biomolecular interaction networks", Genome research, vol. 13, no. 11, pp. 2498-2504, 2003, doi: $10.1101 /$ gr.1239303

[6] A. Shimbel, "Structural parameters of communication networks", The bulletin of mathematical biophysics, vol. 15, pp. 501-507, 1953, doi: 10.1007/ BF02476438 